\title{
The X-ray performance of a driftless gas proportional scintillation counter using short shaping-time constants for pulse analysis
}

\author{
D.S. Covita ${ }^{a}$, P.C.P.S. Simões ${ }^{\mathrm{a}}$, L.M.P. Fernandes ${ }^{\mathrm{a}}$, E.D.C. Freitas ${ }^{\mathrm{a}}$, \\ L.F. Requicha Ferreira ${ }^{\mathrm{a}}$, J.F.C.A. Veloso ${ }^{\mathrm{a}, \mathrm{b}}$, J.M.F. dos $\operatorname{Santos}^{\mathrm{a}, *}$ \\ ${ }^{a}$ GIAN, Physics Department, University of Coimbra, P-3004-516 Coimbra, Portugal \\ ${ }^{\mathrm{b}}$ Physics Department, University of Aveiro, P-3810-193 Aveiro, Portugal
}

Received 29 May 2003; accepted 29 July 2003

\begin{abstract}
Performance characteristics are evaluated for a xenon driftless gas proportional scintillation counter (GPSC), for which detector pulses are formatted using very short shaping-time constants $(\sim 50 \mathrm{~ns})$, and directly analyzed by the MCA without previous pulse time duration analysis and amplitude correction. The present detector and method allow the achievement of detector energy linearity and energy resolution similar to those of conventional GPSCs, reducing background levels and maximizing the detector count-rate capability. However, the obtained peak tails can be somewhat larger than those obtained with conventional GPSCs for X-ray energies above $\sim 30 \mathrm{keV}$. Energy resolutions of $7.7 \%, 4.5 \%$ and $3.8 \%$ can be achieved for $5.9,22.1$ and $59.6 \mathrm{keV} \mathrm{X}$-rays, respectively.

(C) 2003 Elsevier B.V. All rights reserved.
\end{abstract}

\section{Introduction}

Gas proportional scintillation counters (GPSCs) became established detectors in X-ray astronomy applications (see Ref. [1] and references therein). While this has been the most visible purpose of these detectors, their application to X-ray fluorescence analysis, X-ray structure analysis, medical instrumentation, medium- and high-energy physics have been investigated [2-7]. Their advantages include large area capability, ruggedness, room

\footnotetext{
*Corresponding author. Tel.: + 351-239-410667; fax: + 351239-829158.

E-mail address: jmf@gian.fis.uc.pt (J.M.F.dos Santos).
}

temperature operation, superior energy resolution and higher count-rate capability without spacecharge effects, when compared with other types of gas detectors. Additionally, they can operate under intense magnetic fields [4,5].

Typically, a GPSC consists of a noble-gas volume divided in two regions - the absorption and the scintillation region - and a photosensor (Fig. 1). The preferred gas medium is usually xenon, chosen for its large photoionization crosssection and high scintillation efficiency. X-ray photons are absorbed in the drift region by photoelectric effect and the resulting primary electron cloud drifts towards the scintillation region under the influence of a weak electric field, 


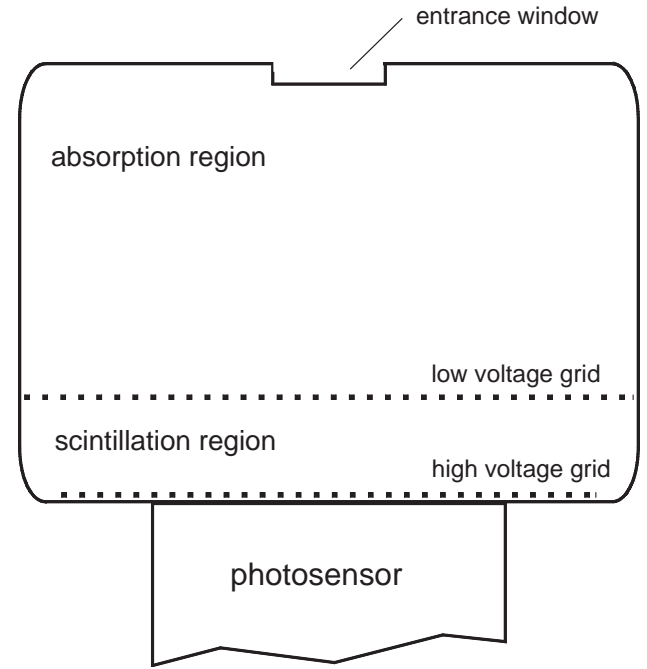

Fig. 1. Schematic diagram of a conventional GPSC.

which is below the gas scintillation threshold. In the scintillation region, however, the electric field is above the gas scintillation threshold, but below the ionization threshold. Upon crossing the scintillation region, the electrons' kinetic energy is high enough to excite but not ionize the gas atoms, giving rise to a large number of VUV photons, as a result of the atoms' de-excitation process, without undergoing charge avalanche amplification. The integral amount of VUV photons collected in the photosensor is proportional to the number of primary electrons crossing the scintillation region and, thus, to the energy of the incident X-ray, independently of the position where the X-ray interaction occurred.

In contrast to charge avalanche processes, the statistical fluctuations associated to the light amplification process are negligible compared to those associated with the formation of the primary electron cloud. Thus, the GPSC energy resolution approaches its intrinsic value, provided that the fluctuations associated to the photosensor are also kept low. GPSCs' energy resolutions are better than those of proportional counters and can be superior to those of solid-state detectors when large detection areas are required. In the sub-keV region, the energy resolution of a GPSC is even better than that obtained with cryogenic solidstate detectors, e.g. see Ref. [8].

However, the interaction of X-rays in a region with a weak electric field is disadvantageous for soft X-rays (typically with energies below $2-3 \mathrm{keV}$ ) with low penetration depths in the gas, since some of the primary electrons may be lost to the detector radiation window, owing to diffusion. This results in distortion of the pulse-height distributions, which depart from the gaussian shape, presenting low-energy tails that extend towards very low amplitudes [8-10]. Additionally, other degrading effects, such as losses of primary electrons to the low-voltage grid that separates the drift and the scintillation regions, losses due to lateral diffusion of the primary electron cloud and losses to impurities, are present in conventional GPSCs, due to the very large number of electron collisions along its path in the drift region [11]. All these effects contribute to detector energy resolution degradation and increased background level. It was shown that these effects are very sensitive to the electric field in the drift region and are reduced with increasing electric field. However, this field should be kept below the gas scintillation threshold, and field-ratios above 5-10 should be used between scintillation and drift regions, in order to keep the electron transmission of the low-voltage grid around $100 \%[11,12]$.

In response, driftless GPSCs were developed [8,13-17], in which the drift region is eliminated and the X-rays are absorbed directly in the scintillation region. Driftless-GPSCs are characterized by an intense electric field throughout the detector volume, significantly reducing primary electron losses and providing, thus, improved energy resolution and background level compared to conventional configurations.

Unlike a GPSC with drift region, the total amount of light produced as a consequence of an $\mathrm{X}$-ray interaction will depend on the distance traversed by the primary electron cloud in the scintillation region, and, hence, on the depth where the interaction occurred. Since the light-pulse duration is proportional to this distance, the timing analysis of driftless GPSC pulses allows pulse amplitude correction to restore proportionality between the corrected pulse and the 
corresponding X-ray photon, independently of its interaction depth. Pulse amplitude correction is unnecessary for soft X-rays with absorption lengths much smaller than the length of the scintillation region. In this case, the effect of the different X-ray penetration depths on the distance covered by the primary electron cloud in the scintillation region and, thus, on the scintillation light produced, is small. Nevertheless, if higher energy X-rays are present, the low-energy peaks will be superimposed on a high-level background that can only be eliminated by using pulse discrimination- and correction techniques. On the other hand, the applicability and advantages of driftless GPSCs extend to higher energy ranges [8,13-17]. Thus, driftless-GPSCs are generally used combined with pulse time analysis and amplitude correction.

To implement the required pulse amplitude and time analysis, and perform amplitude correction, an array of analog electronics [8] or costly digitizers with specific software for digital pulse processing $[13,14,17]$ is needed. However, for some applications, the improved performance of driftless GPSCs more than compensates for the increased electronic complexity. Recently, a very simple technique was described, which eliminates the need for the additional analog or digital pulse time analysis and amplitude correction, with only a slight energy-dependent degradation in the detector performance [18]. The straightforward use of very short linear shaping-time constants ( $\sim 50 \mathrm{~ns})$, in contrast to the long shaping-time (several $\mu$ s) needed to obtain the integral amount of light, enables pulse shapes to closely represent the scintillation light-pulse time profile. Still, this profile presents a maximum that is directly proportional to the number of electrons in the primary electron cloud and, thus, to X-ray energy, independently of its interaction depth.

In the present work, we investigate the X-ray performance of a driftless GPSC using short shaping-time constants for pulse analysis. Detector performance characteristics, as energy resolution, peak shape and background level, will be investigated as a function of electric field and X-ray energy, in the 1-60-keV range, and compared with those obtained with conventional GPSCs.

\section{Experimental set-up and method}

The driftless GPSC used in this work is depicted in Fig. 2. It has a $10-\mathrm{cm}$ diameter $\times 2.5-\mathrm{cm}$ deep scintillation region, and is filled with xenon, which is continuously purified through getters (SAES St707 type) at $150-200^{\circ} \mathrm{C}$, and maintained in circulation by convection. The upper part of the detector body is made of Macor, which insulates the 8 -mm diameter $\times 7.5$ - $\mu \mathrm{m}$ thick Kapton radiation window and its stainless steel holder. The Kapton, the stainless steel and the Macor are epoxied to each other. The Kapton window is aluminized on the inner side to ensure electrical conductivity. The lower part of the detector is built from stainless steel and is connected to the gas circulation tubing. The bottom of the detector is a Macor disc epoxied to the 51-mm diameter PMT and to the detector wall. The upper and lower parts of the detector are vacuum-tight by compression of an indium gasket.

The PMT is a $5.1-\mathrm{cm}$ diameter linear focused EMI D676QB (an 8-dynode version of the EMI 9266QB PMT). A chromium grid ( $\sim 0.1$-mm-wide line with 1-mm spacing) was vacuum deposited on the PMT quartz window and connected to the photocathode pin through a continuous chromium film deposited on the lateral surface, in order to inhibit any voltage difference between the photocathode and the PMT-window external surface. This design enables the PMT to be in direct contact with the scintillation region, eliminating the need for an extra optical window and maximizing the light collected by the PMT. The window holder and the upper Macor piece were

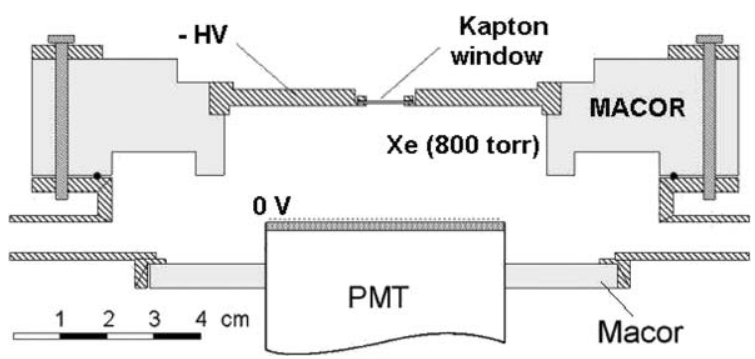

Fig. 2. Schematic diagram of the driftless GPSC used in the present work. 
designed to ensure a uniform electric field in the scintillation region.

The Kapton window and holder are kept at negative high voltage, $-\mathrm{HV}$, while the chromium grid and the PMT photocathode are kept at $0 \mathrm{~V}$. The scintillation region is delimited by the detector radiation window and the PMT window, the electric field in the scintillation region being determined by $-\mathrm{HV}$. The high voltage for the scintillation region biasing does not need to have a low-ripple power supply, while the PMT requires a low-ripple power supply for noise reduction.

Driftless GPSCs have always relied on long integration and differentiation time constants (several $\mu \mathrm{s}$ ) for pulse shaping, in order to measure the total amount of scintillation, prior to correcting the individual pulse amplitudes. By determining the X-ray signal using the total amount of light collected in the photosensor, the statistical fluctuations associated with the light amplification process are minimized. However, the use of very short linear amplifier shaping-time constants ( $\sim 50 \mathrm{~ns}$ ) will provide detector pulses that closely represent the scintillation light-pulse time profile. Fig. 3a shows typical pulse shapes obtained with a $20-\mathrm{MHz}$ digitizer [17]. These selected pulses correspond to $22.1-\mathrm{keV} \mathrm{X}$-ray interactions at different depths. These profiles are similar to those of Ref. [8], obtained with analog electronics. Although the number of scintillation photons produced per electron and per unit of path length is constant at all depths, the number of detected photons in the photosensor increases continuously with depth, due to the increase in the solid angle subtended by the photosensor, achieving a maximum when the electron cloud finally reaches the charge collecting anode.

Fig. $3 \mathrm{~b}$ shows the same pulses aligned by their trailing edges. These pulses illustrate the correlation between absorptions that occur at different depths and their corresponding pulse time duration. They also show that the pulse height and the trailing edges have the same shape for all the pulses, within the statistical fluctuations, since all the primary electron clouds have the same number of electrons and roughly the same dimensions, upon reaching the PMT window. If the pulse maximum is achieved, for every primary electron
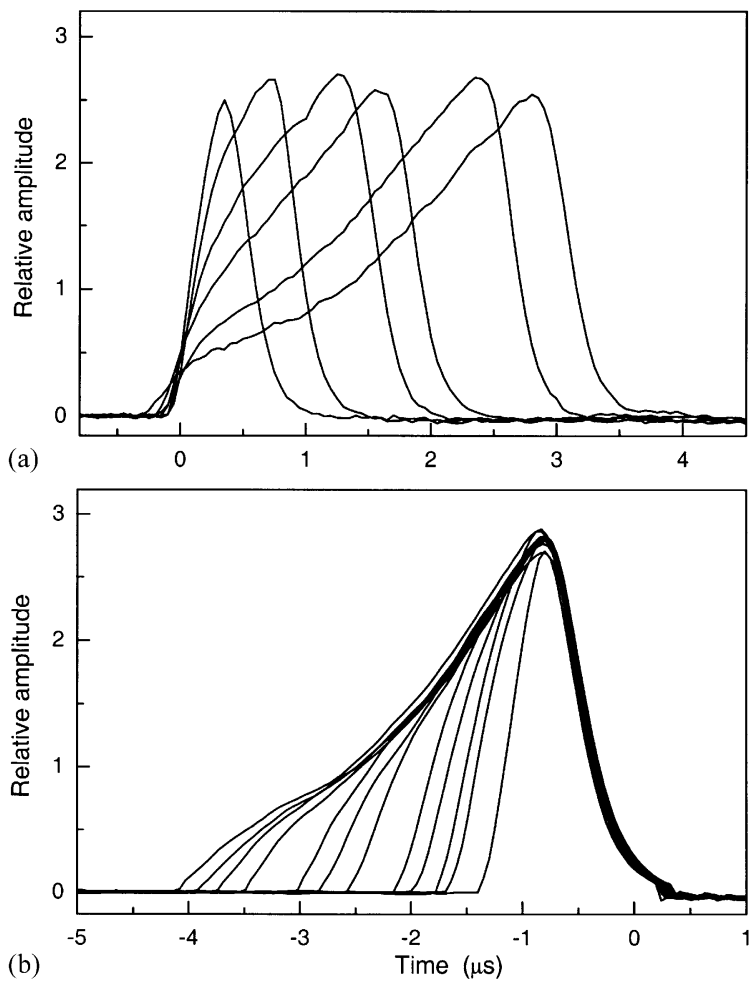

Fig. 3. Typical pulse shapes of the driftless GPSC. The selected pulses correspond to $22.1-\mathrm{keV}$ X-ray interactions at different depths. In (b), the same pulses are aligned by their trailing edges.

cloud, at about the same position, its value depends only on the number of primary electrons present in that cloud, regardless of where the Xray absorption took place and, thus, is proportional to the X-ray energy. The only exceptions are those absorptions occurring at distances closer to the collection anode than the distance for which the maximum detected light occurs.

Thus, the use of short shaping-time constants in driftless GPSCs will provide detector pulses with amplitudes proportional to the X-ray energy, which can be directly pulse height analyzed in a conventional MCA without further corrections. A very good performance for many applications can be achieved with pulse-height analysis using very short shaping times, without further processing and compensation, extending the use of simple, inexpensive driftless-GPSCs-based X-ray spectrometers to the high-energy range [18]. 
The detector pulses were preamplified with an HP5554A charge-to-voltage preamplifier (sensitivity of $10-\mathrm{mV} / \mathrm{pC}$ ), linearly amplified with an HP5582A amplifier (operating with 50-ns differentiation and integration shaping-time constants, without second differentiation) and pulse analyzed with a 1024-multichannel analyzer. For peakamplitude and energy-resolution measurements, pulse-height distributions were fitted to a gaussian superimposed on a step-shape background.

The detector performance was investigated for two different filling pressures, 800 and 1400 Torr $\left(1.06 \times 10^{5}\right.$ and $1.90 \times 10^{5} \mathrm{~Pa}$, respectively $)$.

\section{Experimental results and discussion}

\subsection{Detector performance versus scintillation region biasing voltage}

Fig. 4 depicts the relative pulse amplitude, $A$, and detector energy resolution, $R$, as a function of reduced electric field, $E / p$ (the electric field intensity divided by the gas pressure), for 5.9-, $22.1-$, and $59.6 \mathrm{keV} \mathrm{X}$-rays at a filling pressure of $1.90 \times 10^{5} \mathrm{~Pa}$, and for $22.1 \mathrm{-keV}$ at $1.06 \times 10^{5} \mathrm{~Pa}$. As shown, the relative variation of pulse amplitude with reduced electric field is the same for all the cases. On the other hand, pulse amplitudes obtained for the $1.90 \times 10^{5} \mathrm{~Pa}$ filling are a factor

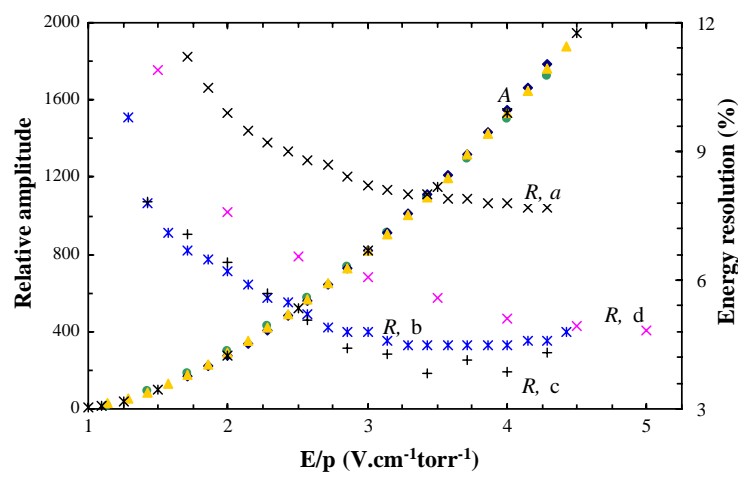

Fig. 4. Detector relative pulse amplitude, $A$, and energy resolution, $R$, as a function of reduced electric field, $E / p$, for: (a) $5.9-\mathrm{keV}$, (b) $22.1-\mathrm{keV}$, (c) $59.6-\mathrm{keV} \mathrm{X}$-rays at $1.90 \times 10^{5} \mathrm{~Pa}$ filling pressure, and (d) $22.1-\mathrm{keV}$ at $1.06 \times 10^{5} \mathrm{~Pa}$. of about two higher than those obtained for the $1.06 \times 10^{5} \mathrm{~Pa}$ filling, at the same conditions.

The experimental results reveal a departure from the approximate linear trend, with a threshold at about $1 \mathrm{~V} \mathrm{~cm}^{-1} \mathrm{Torr}^{-1}$, characteristic of the integral light output measurements (e.g. Ref. [19]). We attribute this behavior to the strong decrease of the primary electron cloud dimensions with increasing reduced electric field, in this range of $E / p$ values. This decrease leads to the fact that the pulse maximum is achieved closer to the PMT with increasing $E / p$, increasing the maximum solid angle subtended by the PMT. In this way, the relative amplitude variation with reduced electric field is faster than the linear variation observed when the total light pulse is integrated.

The detector energy resolution improves with reduced electric field, as the scintillation output increases, presenting the same behavior as obtained for the integral light output measurements [19]. Typically, a fast decrease with increasing $E / p$, at low $E / p$ values and, thus, at low light levels, is observed. The decrease in the energy resolution becomes slower for higher $E / p$ values, as the light level increases and the photosensor statistical fluctuations become much less than the statistical fluctuations associated to the primary electron cloud formation $[19,20]$. The larger light output achieved at higher pressures for the same $E / p$ also explains the better energy resolution obtained for higher pressure fillings.

In this detector, the energy resolution degrades for high biasing voltages (above $13 \mathrm{kV}$ ), due to the increase in the leak current, micro-discharges and electrical instabilities, which arise from the poor electrical insulation of our experimental set-up for those high voltages. These insulation problems are more related with the available SHV connectors and HV cables than with the detector itself. The effect is more significant for high-energy $\mathrm{X}$-rays with lower energy resolutions.

The best energy resolutions obtained with this detector and method were $7.7 \%, 4.5 \%$ and $3.8 \%$ for 5.9-, 22.1- and $59.6 \mathrm{keV}$, respectively. These energy resolutions are similar to those obtained with conventional GPSCs, having a 4-cm thick drift region [21,22]. 


\subsection{Detector performance Versus X-ray energy}

In Fig. 5, the detector relative pulse amplitude and energy resolution are depicted as a function of $\mathrm{X}$-ray energy. The X-ray energies were generated by exciting $\mathrm{K}$-fluorescence lines in selected secondary targets, positioned at $45^{\circ}$ relative to the detector axis, and few centimeters above the radiation window. As shown in Fig. 5, the detector presents very good energy linearity, identical to any conventional GPSC, exhibiting the small discontinuities at the xenon $\mathrm{K}$ and $\mathrm{L}$ absorption edges, characteristic of xenon gaseous detectors [23]. This denotes that the accurate proportionality between detector pulse height and X-ray energy, achieved with GPSCs relying on total lightamount measurement, is held when short shaping-time constants are used for pulse analysis, as claimed in this new method.

The detector energy resolution also presents similar behavior to any conventional GPSC. For low energy $\mathrm{X}$-rays, the energy resolution varies with $E^{-1 / 2}$, departing from this dependence for high X-ray energies, and becoming constant or, eventually, degrading, as the X-ray energy still increases. The energy resolutions were obtained for non-collimated X-ray beams and, thus, are worse than those achieved for well-collimated beams (e.g. $4.5 \%$ and $5.3 \%$ energy resolutions were obtained for 22.1-keV X-rays emitted from a

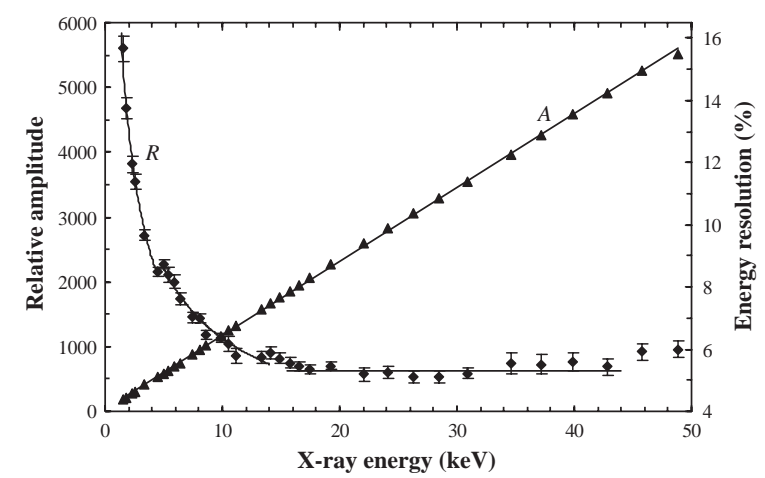

Fig. 5. Detector relative pulse amplitude, $A$, and energy resolution, $R$, as a function of $\mathrm{X}$-ray energy. The solid lines are a linear fit to the amplitude data and $E_{\mathrm{x}}^{-1 / 2}$ fits to the energy resolution data below and above the $\mathrm{Xe}_{\mathrm{L}} \mathrm{III}^{-}$absorption edge, up to $14 \mathrm{keV}$. collimated ${ }^{109} \mathrm{Cd}$ X-ray source and from an $\mathrm{Ag}$ secondary target, respectively).

Similar energy resolutions were obtained in the $1-50-\mathrm{keV}$ range for both the $1.06 \times 10^{5}$ and the $1.90 \times 10^{5} \mathrm{~Pa}$ filling pressure. However, higher pressures allow extending the usefulness of the detector towards the high-energy range. While the pulse-height distribution obtained for the $59.6-\mathrm{keV}$ $\mathrm{X}$-rays presented significant distortions (i.e. large peak tails and high background level) for the $1.06 \times 10^{5} \mathrm{~Pa}$ filling, this degradation was only slight for the $1.90 \times 10^{5} \mathrm{~Pa}$ case (Fig. 6). The obtained energy resolutions are similar to those obtained with a conventional GPSC with a $4-\mathrm{cm}$ thick drift region [22,23] and are only slightly worse than those achieved with the same driftless GPSC, using digital pulse processing techniques for pulse discrimination and correction [18,24], or those achieved with a conventional GPSC with a 7-cm thick drift region [25].

In Fig. 6, we present the pulse-height distributions obtained for ${ }^{241} \mathrm{Am}$ and ${ }^{109} \mathrm{Cd}$ radioactive sources, and for Er-fluorescence X-rays. For comparison, the pulse heights are depicted for different cases: (a) this driftless detector with $1.90 \times 10^{5} \mathrm{~Pa}$ filling, (b) conventional GPSC with a 4 -cm thick drift region and $1.06 \times 10^{5} \mathrm{~Pa}$ filling and (c) conventional GPSC with a 7-cm thick drift region and $1.06 \times 10^{5} \mathrm{~Pa}$ filling. For ${ }^{109} \mathrm{Cd}$, the pulse-height distribution obtained with this detector, using a $1.06 \times 10^{5} \mathrm{~Pa}$ filling, is also included (d). As shown, with the driftless detector only a small degradation in the pulse-height distribution is observed. The peak tails of the $59.6-\mathrm{keV}$ and $\mathrm{Er}$ $\mathrm{K}$-fluorescence $\mathrm{X}$-rays are somewhat higher for the driftless detector. However, the peak-to-background level in the lower part of the pulse-height distribution, after the peak tails, is about the same in the driftless and in conventional detectors. For lower energies, like 22.1-keV X-rays, the peak tails of the driftless detector are already similar to those of conventional detectors, even in the cases when the xenon mass density of the driftless detector is less than that of the conventional GPSC. In fact, the $1.90 \times 10^{5} \mathrm{~Pa}$ driftless detector presents the least background level.

For this method, the amount of scintillation light used to determine the $\mathrm{X}$-ray pulse height is 

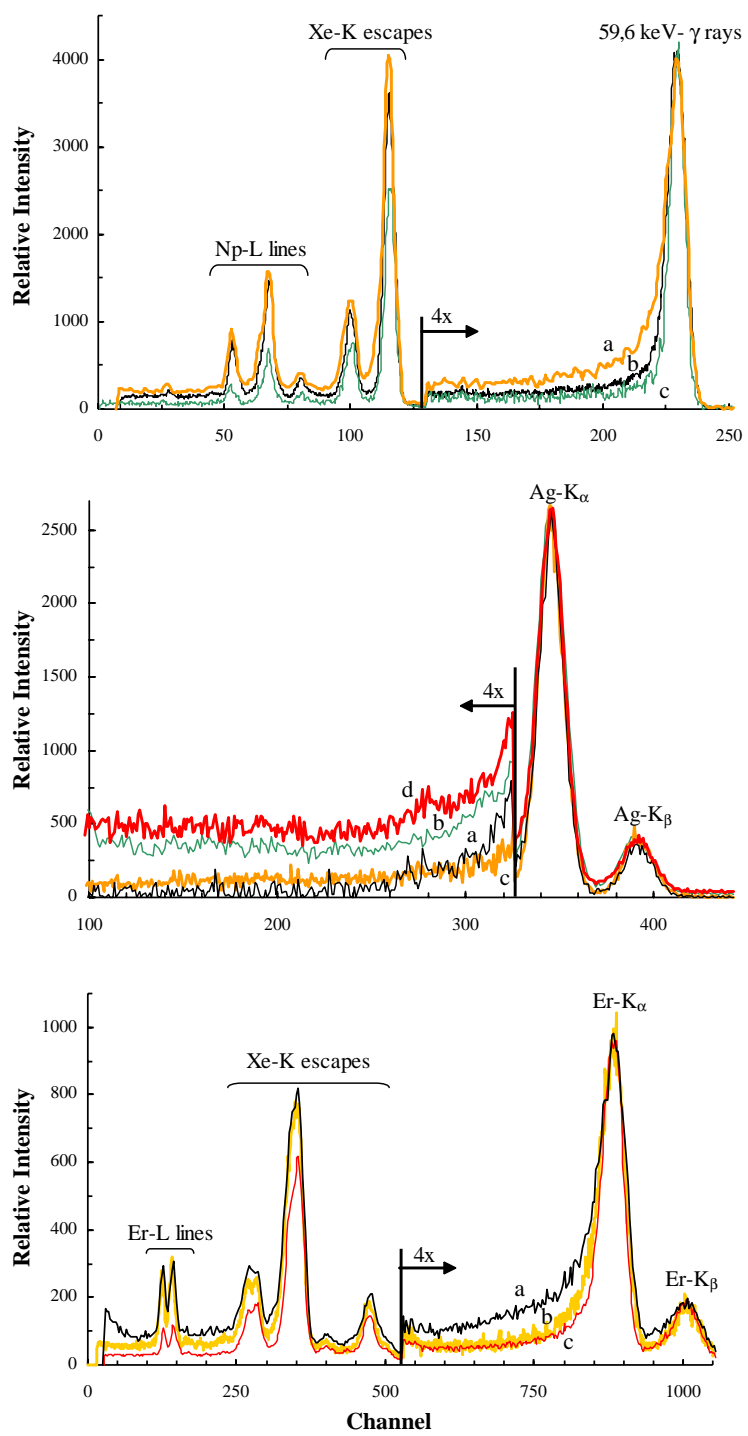

Fig. 6. Pulse-height distributions obtained for ${ }^{241} \mathrm{Am}$ and ${ }^{109} \mathrm{Cd}$ radioactive sources, and for Er-fluorescence X-rays: (a) driftless detector with $1.90 \times 10^{5} \mathrm{~Pa}$ filling, (b) conventional GPSC with a 4-cm thick drift region and $1.06 \times 10^{5} \mathrm{~Pa}$ filling, (c) conventional GPSC with a 7 -cm thick drift region and $1.06 \times 10^{5} \mathrm{~Pa}$ filling and (d) driftless detector using $1.06 \times 10^{5} \mathrm{~Pa}$ filling.

less than when the total area under the pulse profile is considered, i.e. when long integration shaping-time constants are used to determine the detector pulse height. In fact, pulse amplitudes are reduced by a factor of about 10. Thus, the corresponding statistical fluctuations, namely those associated with the photosensor, are somewhat higher and may result in a degradation of the obtained energy resolution, when compared to that obtained when the total light pulse is integrated. This is more important and, eventually, becomes dominant for soft X-rays.

Fig. 7 depicts the pulse-height distributions obtained with the driftless GPSC using $5-\mu$ s and 50-ns shaping-time constants, for X-rays emitted from an aluminum secondary target excited with ${ }^{55} \mathrm{Fe}$ X-rays. Additionally, the pulse-height distribution obtained with a conventional GPSC, having a 4-cm thick drift region, is also depicted in Fig. 7, for comparison. As observed, the least peak tails are obtained with the driftless detector operating at 50-ns shaping-time constants, and both energy resolution and background level of the $\mathrm{Al} \mathrm{K}$-line are similar for either 50 -ns or $5-\mu \mathrm{s}$ shaping-time constants. This demonstrates that the use of the present method delivers similar and/ or better performance, when compared to that obtained by integrating the total amount of light, down to X-ray energies of at least $1 \mathrm{keV}$. Fig. 7 also shows the superior performance of driftless over conventional GPSCs in the soft X-ray region: while the background level is about the same in the region between the $\mathrm{Ca}$ and $\mathrm{Ti} \mathrm{K}$-lines for both types of detectors, the background level of the conventional GPSC increases towards the lower energies, when compared to the driftless detector.

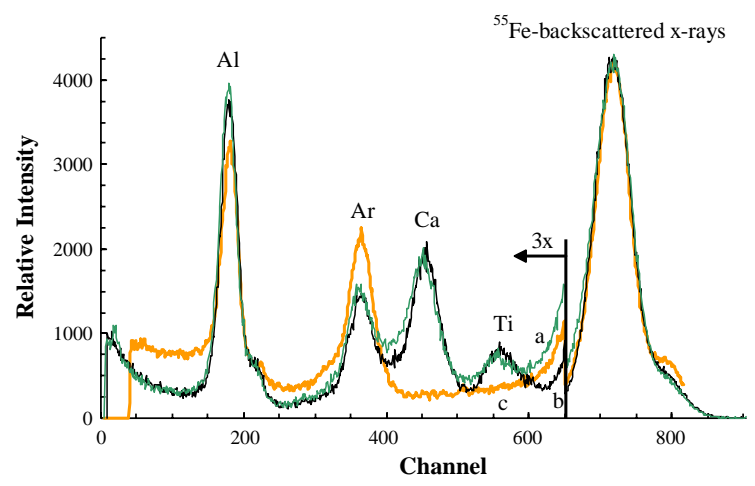

Fig. 7. Pulse-height distributions obtained for X-rays emitted from an aluminum secondary target excited with ${ }^{55} \mathrm{Fe} \mathrm{X}$-rays: (a) driftless GPSC using 5- $\mu$ s shaping time constants, (b) driftless GPSC using 50-ns shaping-time constants and (c) conventional GPSC with 4-cm thick drift region. 
The use of very short shaping-time constants will provide detector pulses that closely represent the temporal development of the scintillation-light pulse. Thus, detector pulse duration is reduced to its intrinsic value, maximizing the detector countrate capability. The maximum light-burst duration is around 4-3- $\mu \mathrm{s}$, for $E / p$ of $4-6 \mathrm{~V} \mathrm{~cm}^{-1} \mathrm{Torr}^{-1}$, resulting in a count-rate capability up to about $25-35 \mathrm{kHz}$ [1]. This value is more than a factor of 5 better than for the case when 5 - $\mu$ s shapingtime constants are used, and more than one order of magnitude higher than what can be achieved when performing pulse time analysis and amplitude correction $[8,17]$.

\subsection{X-ray fluorescence spectrometry performance}

Three examples of pulse-height distributions of fluorescence X-rays from different samples are presented in Fig. 8: non-homogeneous geological samples of anthracite and chalcopyrite excited with an ${ }^{55} \mathrm{Fe}$ and a ${ }^{109} \mathrm{Cd} \mathrm{X}$-ray source, respectively, and a homogeneous sample of SAES St 707 getter $(70 \% \mathrm{Zr}, 5.4 \% \mathrm{Fe}$ and $26.4 \% \mathrm{~V})$ excited with a ${ }^{109} \mathrm{Cd}$ source. The spectral features include the $\mathrm{Pb}$ L-lines emitted from the collimators of the $\mathrm{X}$-ray source and the detector window.

A good performance is achieved for both the low- and the high-energy X-ray range, with reduced peak tails. Energy resolutions of $11.4 \%$, $9.7 \%, 9.2 \%, 7.6 \%, 6.6 \%, 5.5 \%$ and $5.6 \%$ were obtained for $\mathrm{S}, \mathrm{Ca}, \mathrm{V}, \mathrm{Fe}, \mathrm{Cu}$ and $\mathrm{Zr} \mathrm{K}_{\alpha}$-lines, and $\mathrm{Pb}-\mathrm{L}_{\alpha}$-line, respectively. Peaks of $\mathrm{Z}$ and $\mathrm{Z}+2$ elements are completely separated and the $\mathrm{K}_{\alpha^{-}}$and $\mathrm{K}_{\beta}$-lines are partially separated for energies above $6 \mathrm{keV}$.

\section{Conclusions}

We have characterized the performance of a driftless GPSC using very short shaping-time constants $(\sim 50 \mathrm{~ns})$ for pulse formatting. This method enables a straightforward analysis of the detector pulses, directly with the MCA, without previous pulse time duration analysis and amplitude correction, as is currently used. For a xenon depth of $2.5 \mathrm{~cm}$ and a filling pressure of
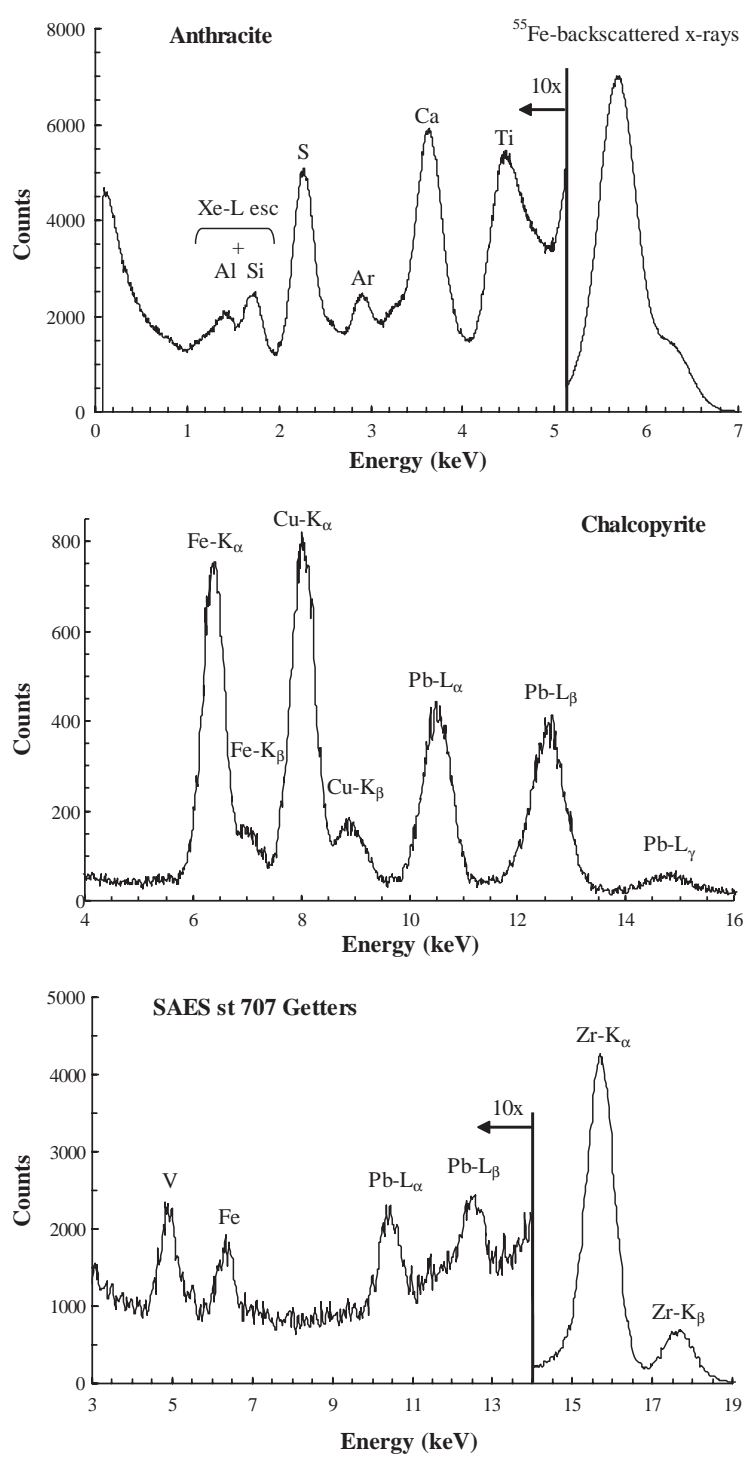

Fig. 8. Pulse-height distributions of fluorescence X-rays obtained for non-homogeneous geological samples of anthracite and chalcopyrite excited with a ${ }^{55} \mathrm{Fe}$ and a ${ }^{109} \mathrm{Cd} \mathrm{X}$-ray source, respectively, and for an homogeneous sample of SAES St 707 getter $(70 \% \mathrm{Zr}, 5.4 \% \mathrm{Fe}$ and $26.4 \% \mathrm{~V})$ excited with a ${ }^{109} \mathrm{Cd}$ source.

$1.06 \times 10^{5} \mathrm{~Pa}$, a good performance is achieved for $\mathrm{X}$-ray spectrometry in the $1-50 \mathrm{keV}$ range. This range can be extended to above $60 \mathrm{keV}$ for a filling pressure of $2 \times 10^{5} \mathrm{~Pa}$. Energy resolutions of $7.7 \%$, $4.5 \%$ and $3.8 \%$ can be achieved for 5.9-, 22.1- and $59.6 \mathrm{keV}$ X-rays, respectively. 
The present detector and method allow achieving detector energy linearity and energy resolutions similar to those of conventional GPSCs, as well as reduced background levels. Additionally, the use of this method maximizes the detector count-rate capability. However, peak tails can be somewhat higher than those obtained with conventional GPSCs in the upper part of the X-ray energy range. It is expected that improved performance will be obtained for higher energies, using higher-pressure fillings and/or larger scintillation depths. For soft X-rays, at least down to $1 \mathrm{keV}$, this method presents a performance similar to that obtained when using $5 \mu$ s time constants.

\section{Acknowledgements}

This work has been carried out in the Instrumentation Centre (unit 217/94) of the Physics Department, University of Coimbra. Fundação para a Ciência e a Tecnologia (FCT) is acknowledged for support through project POCTI/FIS/ 43527/99. J.F.C.A. Veloso acknowledges funding from FCT.

\section{References}

[1] A. Smith, M. Badvaz, Rev. Sci. Instr. 63 (1992) 689.

[2] J.M.F. dos Santos, A.J.V.D. Soares, C.M.B. Monteiro, R.E. Morgado, C.A.N. Conde, IEEE Trans. Nucl. Sci. NS-45 (1998) 229.

[3] D.A. Goganov, A.A. Shultz, Nucl. Instr. and Meth. A 394 (1997) 151.

[4] J.F.C.A. Veloso, J.M.F. dos Santos, C.A.N. Conde, F. Mullauser, P.B. Knowles, C. Donche-Gay, O. Huot, D. Taqqu, F. Kottmann, Nucl. Instr. and Meth. A 460 (2001) 297.

[5] J.F.C.A. Veloso, J.A.M. Lopes, C.A.N. Conde, L.M.P. Fernandes, E.D.C. Freitas, O. Huot, P.B. Knowles, F. Kottmann, F. Mullauser, J.M.F. dos Santos, D. Taqqu, IEEE Trans. Nucl. Sci. NS-49 (2002) 899.

[6] L. Periale, V. Peskov, P. Carlson, T. Francke, N. Pavlopoulos, P. Picchi, F. Pietropaolo, Detection of the various primary scintillation light from dense $\mathrm{Ar}, \mathrm{Kr}$, and
Xe with novel photosensitive gaseous detectors, Physics/ 0106048, 15 June, 2001.

[7] L. Periale, V. Peskov, P. Carlson, C. Iacobeause, T. Francke, N. Pavlopoulos, F. Pietropaolo, T. Sokolova, P. Picchi, Nucl. Instr. and Meth. A 497 (2003) 242.

[8] D.G. Simons, P.A.J. De Korte, Nucl. Instr. and Meth. A 277 (1989) 642.

[9] H. Inoue, K. Koyama, M. Matsuoka, T. Ohashi, Y. Tanaka, H. Tsunemi, Nucl. Instr. and Meth. A 157 (1978) 295.

[10] F.P. Santos, J.M.F. dos Santos, T.H.V.T. Dias, C.A.N. Conde, IEEE Trans. Nucl. Sci. NS-42 (1995) 611.

[11] D.G. Simons, P.A.J. Korte, A. Peacock, J.A.M. Bleecker, Proc. SPIE 597 (1985) 190.

[12] F.I.G.M. Borges, C.A.N. Conde, in: T.D. Mark, R. Schrittwieser, D. Smitt (Eds.), Proceedings of SASP 94, Hintermoos, Austria, Institute für Ionen Physic, Universität Innsbruck, 20-24 March, 1994, p. 436.

[13] A. Smith, A. Peacock, T.Z. Kowalski, IEEE Trans. Nucl. Sci. NS-34 (1987) 57.

[14] A. Smith, F. Favata, T.Z. Kowalski, Nucl. Instr. and Meth. A 284 (1989) 375.

[15] M. Barbera, A. Collura, F. Favata, G. Inzerillo, F. Mirabello, Proc. SPIE 2280 (1994) 154.

[16] M. Bavdaz, A. Peacock, A.N. Parmar, D. Fuchs, P. Müller, F. Scholze, G. Ulm, A.C. Wright, Nucl. Instr. and Meth. A 345 (1994) 549.

[17] P.C.P.S. Simões, J.M.F. dos Santos, C.A.N. Conde, X-ray Spectrom. 30 (2001) 342.

[18] P.C.P.S. Simões, D.S. Covita, J.F.C.A. Veloso, J.M.F. dos Santos, R.E. Morgado, A new method for pulse analysis of driftless proportional scintillation counters, Nucl. Instr. and Meth. A 505 (2003) 247.

[19] F.I.G.M. Borges, J.M.F. dos Santos, T.H.V.T. Dias, F.P. Santos, P.J.B.M. Rachinhas, C.A.N. Conde, Nucl. Instr. and Meth. A 422 (1999) 321.

[20] J.F.C.A. veloso, J.M.F. dos Santos, C.A.N. Conde, Nucl. Instr. and Meth. A 457 (2001) 253.

[21] J.M.F. dos Santos, A.C.S.S.M. Bento, C.A.N. Conde, Nucl. Instr. and Meth. A 337 (1994) 427.

[22] J.M.F. dos Santos, J.F.C.A. Veloso, R.E. Morgado, C.A.N. Conde, Nucl. Instr. and Meth. A 353 (1994) 195.

[23] T.H.V.T. Dias, J.M.F. dos Santos, P.J.B.M. Rachinhas, F.P. Santos, C.A.N. Conde, A.D. Staufer, J. Appl. Phys. 82 (1997) 2742.

[24] P.C.P.S. Simões, J.M.F. dos Santos, C.A.N. Conde, X-ray Spectrom. 30 (2001) 342.

[25] H. Natal da Luz, J.F.C.A. Veloso, J.M.F. dos Santos, C.A.N. Conde, R.M.C. Silva, H.-R. Pan, Z.-Y. Li, H.-A. Lin, IEEE Trans. Nucl. Sci. NS-49 (2002) 2488. 